\title{
Article
}

\section{Single-Molecule Solvation-Shell Sensing}

Leary, E., Höbenreich, H., Higgins, S., van Zalinge, H., Haiss, W., Nichols, R., Finch, C., Grace, I., Lambert, C., McGrath, R. and Smerdon, Joe

Available at http://clok.uclan.ac.uk/9490/

Leary, E., Höbenreich, H., Higgins, S., van Zalinge, H., Haiss, W., Nichols, R., Finch, C., Grace, I., Lambert, C. et al (2009) Single-Molecule Solvation-Shell Sensing. Physical Review Letters, 102 (8). ISSN 0031-9007

It is advisable to refer to the publisher's version if you intend to cite from the work. http://dx.doi.org/10.1103/PhysRevLett.102.086801

For more information about UCLan's research in this area go to http://www.uclan.ac.uk/researchgroups/ and search for <name of research Group>.

For information about Research generally at UCLan please go to http://www.uclan.ac.uk/research/

All outputs in CLoK are protected by Intellectual Property Rights law, including Copyright law. Copyright, IPR and Moral Rights for the works on this site are retained by the individual authors and/or other copyright owners. Terms and conditions for use of this material are defined in the policies page.

\section{CLoK}

Central Lancashire online Knowledge www.clok.uclan.ac.uk

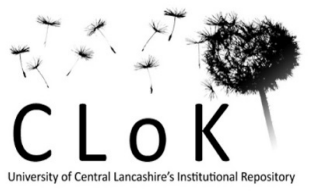




\title{
Single-Molecule Solvation-Shell Sensing
}

\author{
E. Leary, ${ }^{1}$ H. Höbenreich, ${ }^{1}$ S. J. Higgins, ${ }^{1}$ H. van Zalinge, ${ }^{1}$ W. Haiss, ${ }^{1}$ R. J. Nichols, ${ }^{1}$ C. M. Finch, ${ }^{2}$ I. Grace, ${ }^{2}$ \\ C. J. Lambert, ${ }^{2}$ R. McGrath, ${ }^{3}$ and J. Smerdon ${ }^{3}$ \\ ${ }^{1}$ Department of Chemistry, Liverpool University, Liverpool, L69 7ZD, United Kingdom \\ ${ }^{2}$ Department of Physics, Lancaster University, Lancaster, LA1 4YB, United Kingdom \\ ${ }^{3}$ Department of Physics, Liverpool University, Liverpool, L69 7ZE, United Kingdom
}

(Received 14 July 2008; published 23 February 2009)

\begin{abstract}
We present a new route to single-molecule sensing via solvation shells surrounding a current-carrying backbone molecule. As an example, we show that the presence of a water solvation shell "gates" the conductance of a family of oligothiophene-containing molecular wires, and that the longer the oligothiophene, the larger is the effect. For the longest example studied, the molecular conductance is over 2 orders of magnitude larger in the presence of a shell comprising just 10 water molecules. A first principles theoretical investigation of electron transport through the molecules, using the nonequilibrium Green's function method, shows that water molecules interact directly with the thiophene rings, significantly shifting transport resonances and greatly increasing the conductance. This reversible effect is confirmed experimentally through conductance measurements performed in the presence of moist air and dry argon.
\end{abstract}

DOI: 10.1103/PhysRevLett.102.086801

PACS numbers: 73.63.- b, 81.07.Nb, 85.35.-p, 85.65.+h

Single-molecule electronic devices formed from current-carrying backbone molecules, in contact with metallic electrodes are widely recognized as a potential route to sub-10 nm electronics. [1-4]. Although it is now possible to measure the electrical conductance of nanoscale junctions in which a small integer number of molecules bridges two metal contacts [5-11], the realization of scalable architectures on such length scales remains a longterm challenge. Therefore it is of interest to identify intermediate spin-offs, which could be realized on a shorter time scale. In this Letter, we examine one such spin-off, namely, a novel route to reversible, room-temperature single-molecule sensors [12]. For room-temperature operation, the binding energy of a "guest" molecule to a "host" molecule should be larger than $k_{B} T$. On the other hand, for the sensor to be reversible, the binding energy should be not much larger than this, which rules out covalent bonding of a guest to the backbone and instead points to gentler attachment routes such as hydrogen bonding and dipoledipole interactions. When a long rigid host molecule is surrounded by a gas or liquid of smaller guest molecules, such interactions are known to lead to the formation of one or more solvation shells surrounding the host. In this Letter, we demonstrate that the formation of a such a shell can dramatically affect electron transport along the host.

For simplicity, we demonstrate the single-molecule detection of a solvation shell formed from water. This is a remarkable result, since many families of backbone molecules, such as alkanedithiols exhibit the same molecular conductance whether measured in air, under vacuum, or under liquids of different polarity and capacitive sensors based on carbon nanotubes [13] are insensitive to water. Here we show that the presence of water "gates" the conductance of a family of oligothiophene-containing mo- lecular wires, and that the longer the oligothiophene, the larger is the effect. For the longest example studied, the molecular conductance is over 2 orders of magnitude larger in the presence of water, an unprecedented result suggesting that ambient water is a crucial factor to be taken into account when measuring single-molecule conductances, or in the design of future single-molecule electronic devices.

The molecular backbones investigated are shown in Fig. 1(a) and comprise $n+1$ thiophene rings $(n=0,1$, $2,4)$ connected via thiol-terminated alkane chains to gold electrodes. Since the frontier orbitals of the alkyl groups are far from the Fermi energy of the contacts, while the frontier orbitals of the $\pi$-conjugated units are closer to the Fermi energy, these molecules may be thought of as molecular analogues of tunnel-barrier indentations [14,15], with larger barriers formed from the alkane chains and a barrier indentation formed from the thiophene chain. Although one cannot rule out alternative explanations based on incoherent hopping, in what follows, we present a theoretical investigation of electron transport through these molecules, using the first principles nonequilibrium Green's function (SMEAGOL) method [16,17]. We show that water molecules can interact with the thiophene rings, forming a solvation shell which significantly shifts electron transport resonances and greatly increases conductance. We also compare these predictions with detailed measurements of the electrical conductance under ambient conditions and in the presence of dry argon.

Before discussing details of the investigation, we first summarize our key results, which are presented in Fig. 1(d). Since the Fermi level of gold is predicted to lie in the HOMO-LUMO gap, the molecular conductances of oligothiophene molecules are expected to decrease exponentially with the length of the molecule and indeed for 

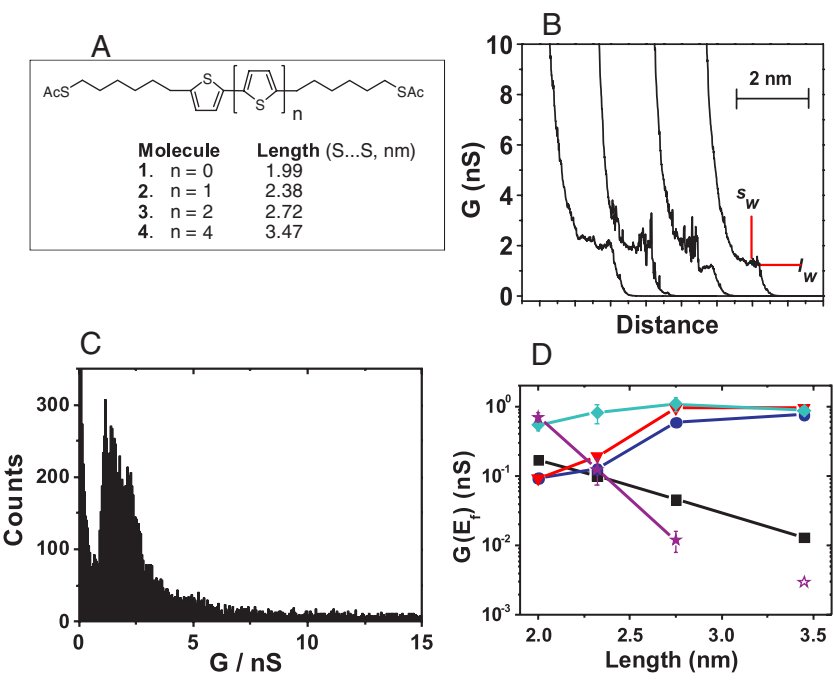

FIG. 1 (color online). (a) Structures of molecules 1-4 used in this study, and the SS distances for the relaxed, fully extended molecules, calculated using molecular mechanics (Spartan04). (b) Example current-distance relations for several $I(s)$ determinations on molecule 2. (c) Histogram of all 72 of the characteristic current plateaus $[I(w)]$ observed for 2 measured in ambient air [set-point current $I(w)=4 \mathrm{nA}$, tip-sample bias $200 \mathrm{mV}$ ]. (d) Experimentally determined single-molecule conductance values (log scale; plotted against molecular length) for 1-4 measured in ambient conditions (light-blue diamonds), computed zero-bias conductance, in the absence of water (black squares) and in the presence of individual water molecules located at the favored "side" positions (red triangles). The blue circles show the computed ensemble-averaged conductance in the presence of side molecules together with randomly positioned background water molecules. Finally, the purple stars show the single-molecule conductances measured under an atmosphere of dry argon (the hollow star for 5 represents an upper limit, since this molecule had a conductance in the absence of water that was outside the range of our current amplifier). It is worth noting that as expected, DFT underestimates the HOMOLUMO gap of these molecules and therefore in the absence of water, the computed length dependence is weaker than the measured behavior. For comparison Tao [33] measures the HOMO-LUMO gap to be $3.4 \mathrm{eV}$ for the 3 ring thiophene and $3.0 \mathrm{eV}$ for the four ring thiophene, whereas our DFT calculation yields 2.15 and $1.64 \mathrm{eV}$, respectively.

molecules $1-4$ of Fig. 1(a), our ab initio theoretical results [black line and squares in Fig. 1(d)] confirm this expectation. In contrast, we also predict that under ambient conditions, where a nm-thick film of water is present on the gold surface $[18,19]$, a solvation shell of water molecules forms around the thiophene backbone, which electrostatically gates the host molecule, shifts the molecular resonances, and increases the electron transmission coefficient. This effect is more pronounced for the longer molecules and cancels the otherwise exponential decay [dark blue curve with circles and red curve with triangles in Fig. 1(d)]. For the longest molecule, we predict that the presence of a solvation shell of only 10 water molecules increases the electrical conductance by 2 orders of magnitude. Figure 1(d) also shows experimental results for 1-4 measured under ambient conditions, in which the backbone molecules are surrounded by moist air [light-blue curve and diamonds in Fig. 1(d)] and in agreement with the theoretical prediction show an almost length-independent conductance.

To demonstrate that the exponential length dependence is restored by removing water, Fig. 2 shows the effect of purging the experiment on molecule 3 with dry argon. As predicted, this causes a dramatic decrease in the electrical conduction. Furthermore, subsequent introduction of water vapor into the experiment reverses this decrease and restores the conductance to the value measured under ambient conditions.

To understand the details of this remarkable behavior, we now present theoretical results, obtained using the SMEAGOL approach. In these calculations, the relaxed geometry of the oligothiophenes was found using the density functional code SIESTA [20]. A double-zeta plus polarization basis set, Troullier-Martins pseudopotentials [21] to remove core electrons and the Ceperley-Alder [22] LDA description of the exchange correlation, were used. Atomic positions were relaxed until all force components were smaller than $0.02 \mathrm{eV} / \AA$. The molecule was then extended to include surface layers of the gold leads; a layer
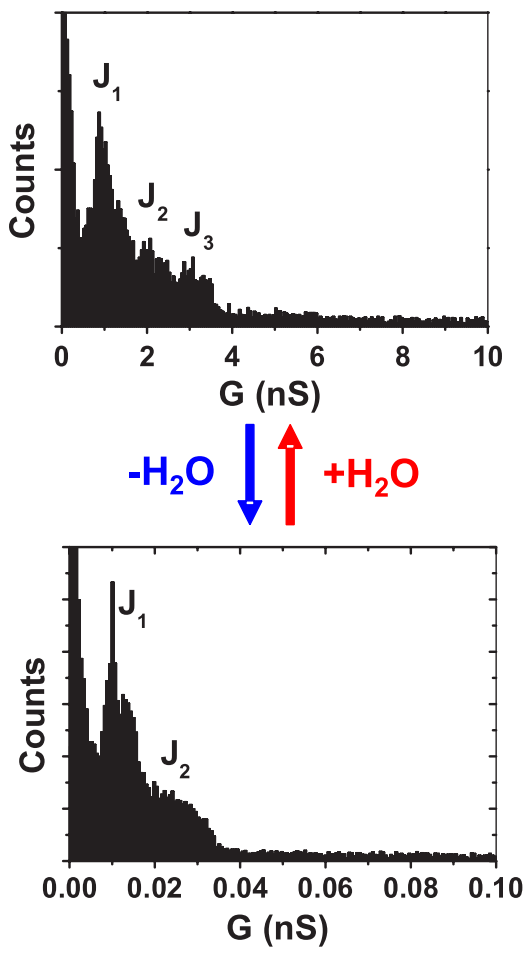

FIG. 2 (color online). Histogram of the characteristic current plateaus $[I(w)]$ observed for molecule 3 measured under (lower) dry argon, $U_{\text {tip }}=+1 \mathrm{~V}$, set point current $=7 \mathrm{nA}$ (upper) after subsequent readmission of ambient (wet) air to the STM chamber, same conditions. 
comprising 14 atoms was chosen and the extended region included 8 layers of gold to allow a suitable representation of charge transfer effects at a molecule-gold interface. A fixed geometry corresponding to the fully extended, alltrans molecule in the junction was assumed. The location of the thiols was taken to be a top site (i.e., directly above a surface gold atom).

The black curves in Figs. 3(a)-3(d) show the resulting electron transmission coefficient $T(E)$ for electrons of energy $E$ for 1-4. These molecules exhibit a decrease in the gap between the HOMO and LUMO resonances as the molecule becomes longer. Despite this increase, Fig. 1(d) (black squares) shows that the conductance of 1-4 decreases exponentially with length. To understand the effect of ambient air, containing water vapor, we undertook detailed $a b$ initio calculations in the presence of surrounding water molecules. It is known that electron transport in a conductance gap can be increased by the presence of random scatterers $[23,24]$. We therefore examined both the relative effects of water molecules in the first solvation shell of the oligothiophene units, occupying fa-

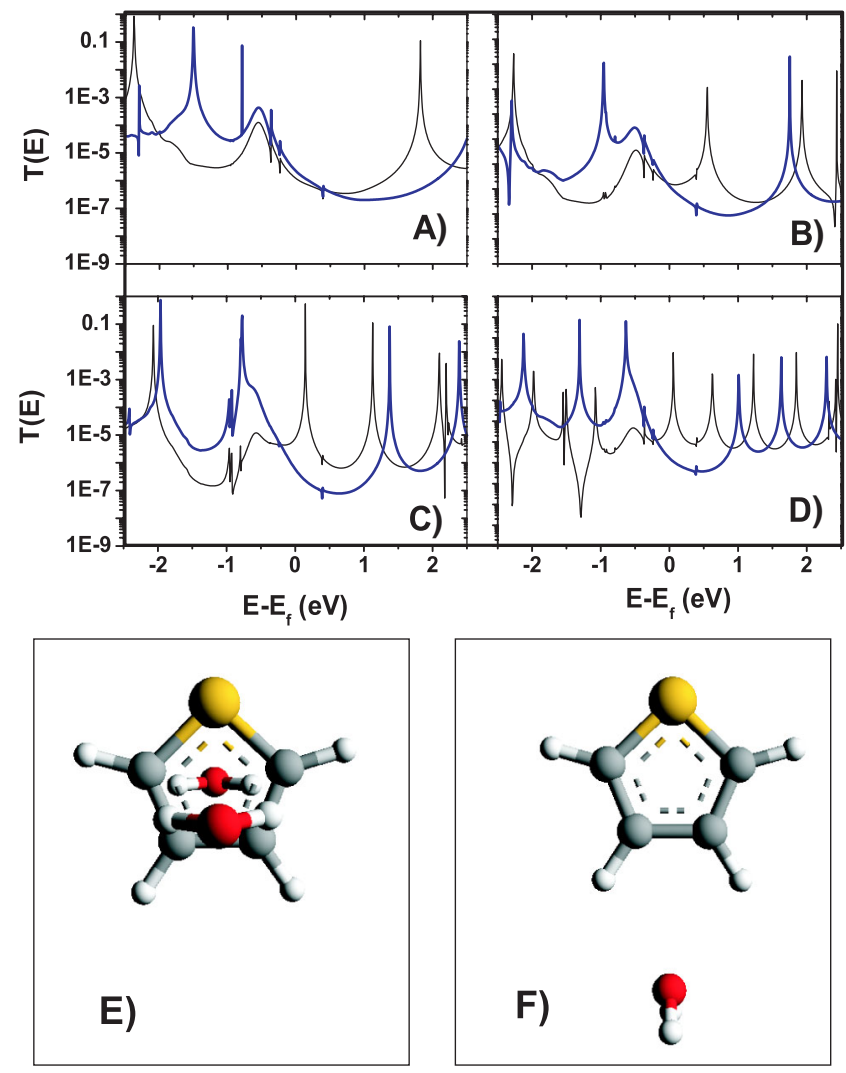

FIG. 3 (color online). Zero-bias transmission coefficient versus energy for 1 (a), 2 (b), 3 (c), and 4 (d). The blue (or gray) curves are obtained in the absence of water and the black curves in the presence of two water molecules located at the lowest energy side positions as illustrated in (e). Water can also interact as shown in the bottom site (f), although the binding energy is lower. vored local energy minima positions, and the effects of randomly positioned water molecules beyond the first solvation shell.

First we performed molecular dynamics simulations to find the optimum location of a water molecule in the vicinity of the thiophene units. For molecule 1, Fig. 3 shows the location of two local minima corresponding to positions, which we call "side" [Fig. 3(e)] and "bottom" [Fig. 3(f)]. The side position is the lowest energy position (in qualitative agreement with previous work [25]) and it also produces the most dramatic change in the conductance, and therefore we initially recalculated the conductance in the presence of side molecules alone. For 1-4, we include two side water molecules per thiophene ring. Figures 3(a)-3(d) show that in the presence of these "side" molecules, the transmission resonances are significantly shifted with respect to their vacuum positions. In particular, the LUMO resonance shifts towards the Fermi energy in all molecules, thus causing an increase in the zero-bias conductances. Crucially, the longer molecules exhibit greater shifts, due to the presence of a larger number of water molecules, which in turn compensates for the reduction in conductance as a function of length. Figure 1(d) (red triangles) shows the zero-bias conductance in the presence of water molecules in the side configuration. Clearly, the exponential length dependence is removed and, in agreement with experiment, after an initial rise for small $n$ the predicted conductance is almost independent of length. It is interesting to note that a similar shift in transmission resonances is obtained for water in the less energetically favorable bottom position of Fig. 3(f) (not shown here).

We also examined the effect of a homogeneously disordered cloud of water molecules surrounding the molecules, and we find that this alone does not remove the exponential length dependence. However, when included together with the side-bound water, additional homogeneously disordered water does have a small but significant additional effect upon the calculated conductances [Fig. 1(d), blue circles]. Random water also leads to a smearing of transmission resonances, so that in practice, steps in the $I-V$ characteristics associated with Figs. 3(c) or $3(d)$ are not observed.

The above predictions are confirmed by $I(s)$ conductance measurements [26,27] on molecules 1 and 3 under water-free conditions. Samples of molecules 1-4 [1(a)] were synthesized and characterized by standard chemical techniques. In the $I(s)$ method, the tunneling current $(I)$ is measured as the STM tip is withdrawn (distance, $s$ ) and molecular bridges are extended in the STM gap. A plateau current $I(w)$ is seen due to current through the molecular bridge with the molecule fully extended. Results from many experiments are analyzed using current histograms as in Fig. 1. Figures 1(b) and 1(c) show examples of these measurements. Low-coverage monolayers of 1-4 (as di- 
thiolates) on clean, atomically flat gold surfaces were prepared and characterized by $\mathrm{x}$-ray photoelectron spectroscopy. These were used for the $I(s)$ determinations $[8,26]$. Figure 2 compares the conductance histograms for 3 measured after purging the STM sample chamber with dry argon for $24 \mathrm{~h}$ (bottom), and subsequently after readmitting ambient air (top). The conductance of 3 determined under argon is $(0.012 \pm 0.006) \mathrm{nS}$ almost 2 orders of magnitude smaller than the conductance in air. When ambient air was readmitted to displace the argon in the ambient pressure STM chamber (or when the experiment was conducted under Ar in the presence of water vapor), the conductance returned to the value previously found under ambient conditions (within experimental error), $(1.0 \pm 0.2) \mathrm{nS}$.

This result is reproducible. We also undertook experiments to confirm that it is indeed water that affects conductance, and not some other atmospheric component. It is known that oligothiophenes undergo charge transfer interactions with $\mathrm{O}_{2}$ [28]. However, (i) we found that $\mathrm{O}_{2}$ had no effect on the conductance of junctions involving 1-4 in theoretical calculations, and (ii) we measured the conductance of $\mathrm{Au}-$ molecule-Au junctions with 3 and 4 under a water-saturated argon atmosphere, and found the same conductance values as in ambient (moist) air.

We tried to measure the conductance of junctions with molecule 4 under dry Ar, but we could not measure meaningful $I(s)$ data; the conductance of 4 under Ar was too low for the current follower on our STM even with $U_{\text {tip }}=$ $1.0 \mathrm{~V}$. This puts an upper limit of the conductance of 4 in the absence of water of $0.006 \mathrm{nS}$, over 2 orders of magnitude smaller than in the presence of water. In contrast, and again in agreement with the theoretical calculations, the conductance of 1 did not change significantly under argon compared with ambient air.

The observation that water, by weakly interacting with the $\pi$ system of a molecule, can drastically alter the molecular conductance of the gold-molecule-gold junction, has wider implications in other important electron transfer processes. Water is known significantly to affect rates of electron transfer (ET) reactions in biological systems by mediating ET coupling pathways and changing activation free energies [29]. Unusual "structured" water molecules near redox cofactors have been found to accelerate ET kinetics when the cofactors are close together; such water appears to show anomalously weak distance decay for electron tunneling [30]. Similarly, in a family of covalently linked azurin complexes, it was found that a longer linkage between azurin units led to a higher rate constant for intramolecular ET, apparently because the longer linkers permitted a dimer pair of water molecules to play a structural role that allowed a better path for electron transfer [31]. In these two instances, it is believed that water is involved in the electron tunneling pathway, whereas in 3 and 4 it "gates" the electron tunneling pathway through the molecule. It has been suggested that water could interact strongly with thiol-gold contacts [32], leading to a length-independent lowering of the conductance of gold-molecule-gold junctions, for simple alkanethiols and alkanedithiols.

We acknowledge support from EPSRC and the EC ITN FUNMOLS.

[1] C. Joachim, J. K. Gimzewski, and A. Aviram, Nature (London) 408, 541 (2000).

[2] M. A. Reed et al., Science 278, 252 (1997).

[3] A. R. Champagne, A. N. Pasupathy, and D. C. Ralph, Nano Lett. 5, 305 (2005).

[4] Y. Luo et al., Chem. Phys. Chem. 3, 519 (2002).

[5] M. A. Reed et al., Science 278, 252 (1997).

[6] X. D. Cui et al., Science 294, 571 (2001).

[7] B. Q. Xu and N. J. Tao, Science 301, 1221 (2003).

[8] W. Haiss et al., J. Am. Chem. Soc. 125, 15294 (2003).

[9] G. Morales et al., J. Am. Chem. Soc. 127, 10456 (2005).

[10] J. Chen et al., Science 286, 1550 (1999).

[11] N. J. Tao, Nature Nanotech. 1, 173 (2006).

[12] X. Y. Xiao, B. Q. Xu, and N. J. Tao, Angew. Chem. 43, 6148 (2004).

[13] E. S. Snow et al., Science 307, 1942 (2005).

[14] L. L. Chang, L. Esaki, and R. Tsu, Appl. Phys. Lett. 24, 593 (1974).

[15] T. J. Foster et al., Phys. Rev. B 39, 6205 (1989).

[16] A. R. Rocha et al., Phys. Rev. B 73, 085414 (2006).

[17] A. R. Rocha et al., Nature Mater. 4, 335 (2005).

[18] J. Y. Jang, G. C. Schatz, and M. A. Ratner, Phys. Rev. Lett. 92, 085504 (2004).

[19] L. Sirghi, R. Szoszkiewicz, and E. Riedo, Langmuir 22, 1093 (2006).

[20] J. Soler et al., J. Phys. Condens. Matter 14, 2745 (2002).

[21] N. Troullier and J.L. Martins, Phys. Rev. B 43, 1993 (1991).

[22] D. M. Ceperley and B. J. Alder, Phys. Rev. Lett. 45, 566 (1980).

[23] C. J. Lambert, J. Phys. C 17, 2401 (1984).

[24] C. J. Lambert, Phys. Rev. B 29, 1091 (1984).

[25] S. C. Meng, J. Ma, and Y. S. Jiang, J. Phys. Chem. B 111, 4128 (2007)

[26] W. Haiss et al., Langmuir20, 7694 (2004).

[27] W. Haiss et al., J. Phys. Chem. B 111, 6703 (2007).

[28] M. S. A. Abdou et al., Adv. Mater. 6, 838 (1994).

[29] H. B. Gray and J. R. Winckler, Q. Rev. Biophys. 36, 341 (2003).

[30] J. P. Lin et al., Science 310, 1311 (2005).

[31] U.M.C. van Amsterdam et al., Nat. Struct. Biol. 9, 48 (2002).

[32] D. P. Long et al., Nature Mater. 5, 901 (2006).

[33] Tao et al., Nano Lett. 5, 693 (2005). 